\title{
Critical behavior of density of states near Fermi energy in low-dimensional disordered metals
}

\author{
E. Şaşıŏlu, ${ }^{1,2, *}$ S. Çalışkan, ${ }^{2, \dagger}$ and M. Kumru ${ }^{2, \dagger}$ \\ ${ }^{1}$ Institut für Festkörperforschung, Forschungszentrum Jülich, D-52425 Jülich, Germany \\ ${ }^{2}$ Department of Physics, Fatih University, Büyükçekmece, İstanbul TR-34500, Turkey
}

(Received 9 June 2008; revised manuscript received 11 August 2008; published 27 January 2009)

\begin{abstract}
We study the effect of electron-electron interaction on the one-particle density of states (DOS) $\rho^{(d)}(\epsilon, T)$ of low-dimensional disordered metals near Fermi energy within the framework of the finite temperature conventional impurity diagram technique. We consider only diffusive limit and, by a geometric resummation of the most singular first-order self-energy corrections via the Dyson equation, we obtain a nondivergent solution for the DOS at low energies while for higher energies the well-known Altshuler-Aronov corrections are recovered. At the Fermi level $\rho^{(d)}(\epsilon, T=0) \rightarrow 0$, this indicates that interacting disordered two-dimensional and quasi-onedimensional systems are in insulating state at zero temperature. The obtained results are in good agreement with recent tunneling experiments on two-dimensional GaAs/AlGaAs heterostructures and quasi-onedimensional doped multiwall carbon nanotubes.
\end{abstract}

DOI: 10.1103/PhysRevB.79.035123

PACS number(s): 71.10.Pm, 71.23. $-\mathrm{k}, 71.30 .+\mathrm{h}, 72.15 . \mathrm{Rn}$

\section{INTRODUCTION}

In the last three decades, a great deal of progress has been made toward revealing the behavior of electrons in a random potential. Efforts have led to a detailed understanding of the low-temperature properties of the weakly disordered systems, i.e., systems for which $k_{F} l \gg 1$, where $k_{F}$ is the Fermi wave number and $l$ is the elastic mean-free path. ${ }^{1}$ This understanding has been embodied in weak localization theory and disorder enhanced electron-electron (ee) interaction effects. $^{2,3}$ The interplay of $e e$ interaction and random impurity potential on the transport and thermodynamic properties of disordered systems has been studied intensively. In particular, treatment of the problem within perturbation theory leads to the understanding of the anomalous logarithmic decrease in conductivity with decreasing temperature in twodimensional (2D) electron gas, negative magnetoresistance observed in many 2D and three-dimensional (3D) systems, as well as the depression in the density of states (DOS) near the Fermi energy. ${ }^{3-5}$

Extensive experimental and theoretical studies have shown that interaction effects are enhanced by disorder and generally result in a decrease in the DOS near the Fermi level. Studies on the theoretical side have, for the most part, concerned two extreme limits. In the limit of strong disorder, this decrease takes the form of a complete gap in the DOS at the Fermi energy. ${ }^{6-9}$ It is known that this Coulomb gap can turn a highly disordered pure metal into a poorly conducting insulator. In the opposite limit, i.e., the diffusive limit, in a pioneering paper by Altshuler, Aronov, and Lee (AAL), treated the 2D disordered electron problem within the perturbation theory to lowest order in interaction strength. ${ }^{10}$ The authors showed that interaction effects in a 2D disordered metal lead to the development of a logarithmic singularity in the one-particle DOS, $\delta \rho^{(2)}(\epsilon) \sim \ln (|\epsilon| \tau)$, near the Fermi energy $\epsilon_{F}$, where $\tau$ and $\epsilon$ are the impurity scattering time and the energy of the electron measured from the Fermi level, respectively. Such effects become even more stronger in quasi-one-dimensional (1D) disordered metals, $\delta \rho^{(1)}(\epsilon) \sim$ $-(|\epsilon| \tau)^{-1 / 2}{ }^{3}$ Unlike the low-dimensional systems, the quan- tum corrections to the DOS in 3D is rather small, $\delta \rho^{(3)}(\epsilon)$ $\sim \sqrt{|\epsilon| \tau}$, giving rise to cusp at the Fermi energy. ${ }^{11}$ Extension of the AAL theory to the ballistic limit shows that the interaction effects give rise to nontrivial corrections to the corresponding physical properties of the disordered systems also in this regime. ${ }^{12-19}$ The reduction in the one-particle DOS near the Fermi energy results in suppression of the tunneling conductance at small voltages, a phenomenon commonly known as the zero-bias anomaly (ZBA). Several groups have performed tunneling measurements on 3D and quasi-2D systems since early 1980s, and observed the predicted dependence of the tunneling conductance with voltage. ${ }^{20-25}$

Recently the problem of $e e$ interaction in disordered metals received considerable interest due to the discovery of the unexpected metallic state in high-mobility two-dimensional semiconductors by Kravchenko and co-workers, ${ }^{26,27}$ and development of experimental techniques such as time domain capacitance spectroscopy (TDCS). ${ }^{28}$ The existence of a metallic state in 2D with finite conductivity at zero temperature is in conflict with the conventional weak localization theory, which predicts that even negligible amount of disorder in low-dimensional systems $(d \leq 2)$ localizes electrons at sufficiently low temperatures. Thus, in spite of several theoretical proposals, the metallic state in $2 \mathrm{D}$ is one of the puzzling phenomena that is still waiting for an adequate description..$^{29-36}$ On the other hand, TDCS appears to be a very useful technique for detection of the tunneling current in regimes difficult to access by conventional methods, and, thus allows the quantitative comparison of the existing theories with experiments. ${ }^{37-39}$ Using TDCS Chan and co-workers ${ }^{37,38}$ measured the entire voltage dependence of the tunneling conductance of a two-dimensional electron system in a GaAs/AlGaAs heterostructure for different electron densities. The authors observed the expected logarithmic Coulomb anomaly only in the case of a small suppression of the tunneling current. However, for large suppressions corresponding to small electron densities, the functional form of the ZBA vs bias voltage was significantly deviating from the predictions of the AAL theory, especially in the regime of very small voltages. 
The critical behavior of the DOS for $\epsilon \rightarrow 0$ in reduced dimensions which is not accessible within the first-order perturbation theory is of great interest in understanding the lowtemperature transport and thermodynamic properties of the disordered metals. In this respect an initial attempt was made by Finkelstein ${ }^{40}$ using field-theoretic renormalization-group theory, who found that $\rho^{(2)}(\epsilon) \sim \epsilon^{1 / 4}$ as $\epsilon \rightarrow 0$ for $2 \mathrm{D}$ systems. Since then there has been a lot of attempt to study energy and temperature dependences of the DOS around the Fermi level employing different methods. ${ }^{41-50}$ Kopietz $^{47}$ has considered a $2 \mathrm{D}$ system and, by resumming the most singular contributions to the average DOS via a gauge transformation, obtained that $\rho^{(2)}(\epsilon) \sim C|\epsilon| / e^{4}$ for $\epsilon \rightarrow 0$, where $C$ is a dimensionless constant and $e$ is the charge of the electron. Kamenev and Andreev ${ }^{48}$ using Keldysh $\sigma$ model derived a nonperturbative result for the DOS of quasi-2D systems. Rollbühler and Grabert ${ }^{49}$ extended this work to quasi-1D systems including additionally the interelectrode interactions and obtained a nondivergent solution for the DOS at low energies, which recovers the $(|\epsilon| \tau)^{-1 / 2}$ behavior for higher energies. It should be emphasized that, in reduced dimensions in contrast to first-order perturbation theory (AAL theory), all these different methods yield a nondivergent solution for the DOS around Fermi level with a power-law behavior, whereas for higher energies results of AAL theory is recovered.

The aim of the present work is a detailed study of the critical behavior of DOS around Fermi level in lowdimensional disordered metals within the diagrammatic perturbation theory. This technique, in contrast to above mentioned nonperturbative schemes, provides a mathematically clear and transparent framework in studying impurity problems in condensed-matter physics. In the present work we consider only diffusive regime and go beyond the first-order perturbation theory. We show that a geometric resummation of the most singular first-order self-energy corrections via the Dyson equation gives a nondivergent solution for the DOS at low energies while for higher energies the obtained expressions are reduced to the predictions of the AAL theory. At zero temperature in both dimensions the DOS vanishes at the Fermi energy. In spite of good agreement between present approach and above mentioned nonperturbative treatments for the DOS at small corrections (higher energies), an essential difference appears in the asymptotic energy dependence of the DOS. The remaining part of the paper is organized as follows. In Sec. II high-order perturbation corrections to the DOS is calculated and compared with AAL theory. In Sec. III we dwell on the zero-bias anomaly of the tunneling conductivity and make a qualitative comparison of the obtained results with recent tunneling experiments. Section IV gives the conclusions.

\section{HIGH-ORDER PERTURBATION CORRECTIONS TO THE DOS}

As it is well known the main contributions to the physical properties of disordered systems in the weak localization theory are connected with two singularities: the first appears in the diffusion propagator, characterizing an electron-hole
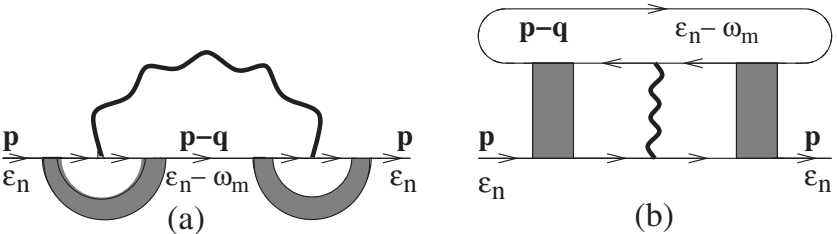

(a)

(b)

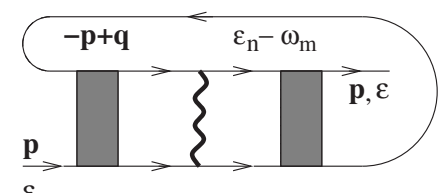

(c)

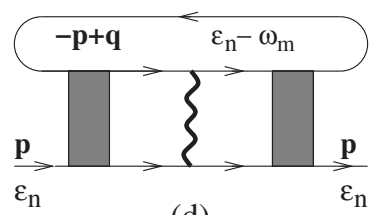

(d)

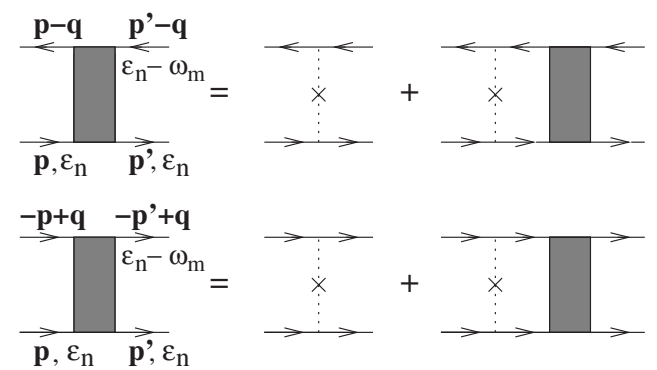

FIG. 1. Diagrams for the calculation of $\Sigma\left(\mathbf{p}, i \epsilon_{n}\right) ;[(\mathrm{a})$ and (c) $]$ exchange diagrams for the diffusion and Cooper channels, respectively; [(b) and (d)] Hartree diagrams for the diffusion and Cooper channels, respectively. The thick wavy lines denote the dynamically screened Coulomb interaction. [(e) and (f)] Ladder series for the diffusion and Cooper channels. Here the dashed line with cross denotes the impurity scattering.

pair with small difference of the momenta $\mathbf{q}$ and of the energies $\omega$ (diffusion pole). The other singularity is due to propagation of electron-electron pair with small sum of the momenta $\mathbf{q}$ and small difference of the energies $\omega$ (Cooper pole). In weak disorder case, corrections to the DOS can be obtained through the self-energy $\Sigma\left(\mathbf{p}, i \epsilon_{n}\right)$. First order in Coulomb interaction contributions to the self-energy are illustrated in Fig. 1. Higher order in Coulomb interaction also gives contributions to the self-energy. Some particular diagrams concerning the second-order self-energy contributions are presented in Fig. 2. However, it can easily be shown that the ratio of the second-order contributions to the first-order

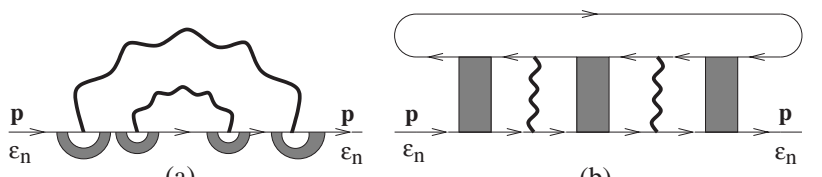

(a)

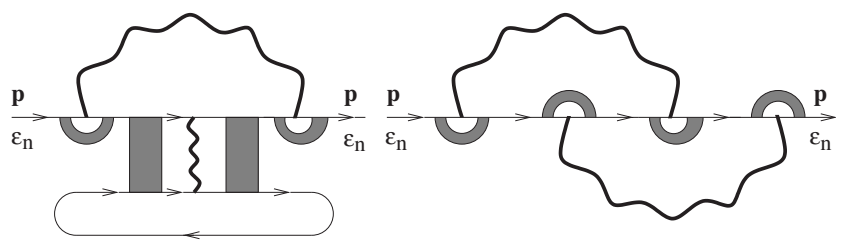

(c)

(d)

FIG. 2. Some particular diagrams for second order in the Coulomb interaction corrections to the self-energy. 
ones is found to be $\lambda_{\rho} \sqrt{|\epsilon| \tau} \ll 1$, where $\lambda_{\rho}$ is the dimensionless constant of interaction. ${ }^{11}$ Therefore we can restrict ourselves to the first-order contributions to the self-energy and neglect small contributions from the higher order selfenergies.

To begin with, consider the exchange interaction between the electrons in the diffusion channel depicted in Fig. 1(a). This process gives rise to a significant contribution to the DOS and the expression for the self-energy can be written as

$$
\begin{aligned}
\Sigma^{D}\left(\mathbf{p}, i \epsilon_{n}\right)= & T \sum_{\omega_{m}} \int \frac{d^{d} q}{(2 \pi)^{d}} V\left(\mathbf{q}, i \omega_{m}\right) G_{0}^{A}\left(\mathbf{p}-\mathbf{q}, i \epsilon_{n}-i \omega_{m}\right) \\
& \times \gamma^{2}\left(\mathbf{q}, i \omega_{m}\right) \theta\left[\epsilon_{n}\left(\omega_{m}-\epsilon_{n}\right)\right]
\end{aligned}
$$

where $G_{0}^{A}$ is the bare temperature Green's function (GF) for electrons averaged over the impurity potential, $\epsilon_{n}=\pi T(2 n$ $+1)$, and $\omega_{m}=2 \pi T m$ are the Matsubara frequencies at temperature T. $\gamma\left(\mathbf{q}, i \omega_{m}\right)$ is the sum of the impurity ladders in Fig. 1, which has a diffusion pole under condition $|\mathbf{q}| l \ll 1$ and $|\omega| \tau \ll 1$. The expression for $\gamma\left(\mathbf{q}, i \omega_{m}\right)$ is given by

$$
\gamma\left(\mathbf{q}, i \omega_{m}\right)=\theta\left[\epsilon_{n}\left(\epsilon_{n}-\omega_{m}\right)\right]+\frac{\theta\left[-\epsilon_{n}\left(\epsilon_{n}-\omega_{m}\right)\right]}{\tau\left(|\omega|+D q^{2}\right)},
$$

with $D=\frac{v_{F}^{2} \tau}{d}$ being the diffusion coefficient for a $d$-dimensional system. $V\left(\mathbf{q}, i \omega_{m}\right)$ in Eq. (1) is the dynamically screened Coulomb potential. Within the random-phase approximation, the $V\left(\mathbf{q}, i \omega_{m}\right)$ takes the following form

$$
\begin{aligned}
V\left(\mathbf{q}, i \omega_{m}\right) & =\frac{2 \pi e^{2}}{|q|+\kappa_{2} \frac{D q^{2}}{|\omega|+D q^{2}}}, \quad(d=2) \\
& =\frac{e^{2}}{e^{2} \rho_{0}^{(1)} \frac{D q^{2}}{|\omega|+D q^{2}}+\ln ^{-1} \frac{1}{q^{2} a^{2}}}, \quad(d=1),
\end{aligned}
$$

with $\kappa_{2}=2 \pi e^{2} \rho_{0}^{(2)}$ being the inverse screening length for a $2 \mathrm{D}$ system, and $a$ is the transverse size of the quasi-1D system. $\rho_{0}^{(d)}$ being the DOS of a noninteracting electron gas which is given by

$$
\rho_{0}^{(1)}=\frac{1}{2 \pi v_{F}}, \quad \rho_{0}^{(2)}=\frac{m}{2 \pi \hbar^{2}}, \quad \rho_{0}^{(3)}=\frac{m p_{F}}{2 \pi^{2} \hbar^{2}} .
$$

Since the $\mathbf{q}$ integral in Eq. (1) is dominated by the diffusive pole of the impurity ladders, then within the accuracy of our calculation, for small $\mathbf{q}$ and $\omega$, Eq. (1) can be rewritten in the following suggestive form:

$$
\Sigma^{D}\left(\mathbf{p}, i \epsilon_{n}\right) \cong \alpha(\epsilon, T) G_{0}^{A}\left(\mathbf{p}, i \epsilon_{n}\right)
$$

with

$$
\alpha(\epsilon, T)=T \sum_{\omega_{m}} \int \frac{d^{d} q}{(2 \pi)^{d}} \gamma^{2}\left(\mathbf{q}, i \omega_{m}\right) V\left(\mathbf{q}, i \omega_{m}\right) .
$$

The DOS $\rho^{(d)}(\epsilon, T)$ of a $d$-dimensional system is defined in terms of the total retarded GF, $G^{R}\left(\mathbf{p}, i \epsilon_{n}\right)$

$$
\rho^{(d)}(\epsilon, T)=-\frac{1}{\pi} \operatorname{Im} \int \frac{d^{d} p}{(2 \pi)^{d}} G^{R}\left(\mathbf{p}, i \epsilon_{n}\right)_{i \epsilon_{n} \rightarrow \epsilon} .
$$

It is well known that the temperature Green's function coincides with the retarded one at discrete points on the positive imaginary semiaxis, i.e., $G\left(\epsilon_{n}\right)=G^{R}\left(i \epsilon_{n}\right)$ at $\epsilon_{n}>0$. According to the Dyson equation the total Green's function $G^{R}\left(\mathbf{p}, i \epsilon_{n}\right)$ including electron correlations in diffusion channel is given by

$$
\begin{aligned}
G^{R}\left(\mathbf{p}, i \epsilon_{n}\right) & =\frac{1}{\left[G_{0}^{R}\left(\mathbf{p}, i \epsilon_{n}\right)\right]^{-1}-\Sigma^{D}\left(\mathbf{p}, i \epsilon_{n}\right)} \\
& =\sum_{n=0}^{\infty}\left[G_{0}^{R}\left(\mathbf{p}, i \epsilon_{n}\right)\right]^{n+1}\left[\Sigma^{D}\left(\mathbf{p}, i \epsilon_{n}\right)\right]^{n} .
\end{aligned}
$$

Substituting Eq. (5) into Eq. (8) and utilizing Eq. (7), the DOS takes the following form ${ }^{51}$

$$
\rho^{(d)}(\epsilon, T)=\rho_{0}^{(d)}-\frac{1}{\pi} \operatorname{Im} \sum_{n=1}^{\infty} A_{n}[\alpha(\epsilon, T)]^{n},
$$

where

$$
A_{n}=\int \frac{d^{d} p}{(2 \pi)^{d}}\left[G_{0}^{R}\left(\mathbf{p}, i \epsilon_{n}\right)\right]^{n+1}\left[G_{0}^{A}\left(\mathbf{p}, i \boldsymbol{\epsilon}_{n}\right)\right]^{n} .
$$

It is easy to see that $n=0$ term in Eq. (9) is equal to $\rho_{0}^{(d)}$; thus the bare DOS is distinguished. Upon performing this integration we find

$$
A_{n}=-\rho_{0}^{(d)} 2 \pi i \tau^{2 n} \frac{n(2 n-1) !}{(n !)^{2}},
$$

Substituting Eq. (11) into Eq. (9) and taking the sum over $n$, one obtains the total contribution to the DOS from the diffusion channel

$$
\rho^{(d)}(\epsilon, T)=\rho_{0}^{(d)}-\rho_{0}^{(d)} \operatorname{Im}\left\{\frac{\beta}{\sqrt{1-i \beta}(1+\sqrt{1-i \beta})}\right\},
$$

where $\beta=4 \tau^{2} \alpha(\epsilon, T)$. Note that we use the expression $\ln [1$ $\left.+\sqrt{1+x^{2}}\right]=\ln 2-\sum_{n=1}^{\infty}(-1)^{n} \frac{(2 n-1) !}{(n !)^{2} 2^{2 n}} x^{2 n}$ in evaluating the sum in Eq. (9).

For a short-range (static) Coulomb interaction, the equation above can be simplified considerably. In this case Coulomb potential depends neither $\mathbf{q}$ nor $\omega$ and thus the integration in Eq. (5) is straightforward, in which calculation for two and one dimensions gives

$$
\begin{aligned}
\alpha(\epsilon, T) & =-\frac{\pi \lambda_{\rho}}{8 \epsilon_{F} \tau^{3}}+i \frac{\lambda_{\rho}}{4 \epsilon_{F} \tau^{3}} \ln \left[\frac{1}{2 \tau(|\epsilon|, T)}\right], \quad(d=2) \\
& =\frac{\lambda_{\rho}}{4 \tau^{2} \sqrt{2 \tau(|\epsilon|, T)}}(1+i), \quad(d=1),
\end{aligned}
$$

where $\lambda_{\rho}=\rho_{0}^{(d)} V(0,0)$ and $\epsilon$ is the energy reckoned from the Fermi level.

If we write $\beta \equiv \beta_{R}+i \beta_{I}$, where $\beta_{R}$ and $\beta_{I}$ are real and imaginary parts, respectively, then Eq. (12) takes the following form 


$$
\rho^{(d)}(\epsilon, T)=\frac{\rho_{0}^{(d)}}{\sqrt{2}} \frac{\sqrt{1+\beta_{R}+\sqrt{\left(1+\beta_{R}\right)^{2}+\beta_{I}^{2}}}}{\sqrt{\left(1+\beta_{R}\right)^{2}+\beta_{I}^{2}}} .
$$

Using Eqs. (13) and (14) one obtains the following expressions for the DOS of 2D and quasi-1D systems:

$$
\begin{gathered}
\rho^{(2)}(\epsilon, T) \simeq \frac{\rho_{0}^{(2)}}{\sqrt{1+\frac{2 \lambda_{\rho}}{\epsilon_{F} \tau} \ln \left[\frac{1}{2 \tau(|\epsilon|, T)}\right]},} \\
\rho^{(1)}(\epsilon, T) \simeq \frac{\rho_{0}^{(1)}}{\sqrt{1+\frac{2 \lambda_{\rho}}{\sqrt{2 \tau(|\epsilon|, T)}}}} .
\end{gathered}
$$

It follows from Eqs. (15) and (16) that for small corrections one recovers the results of the AAL theory:

$$
\begin{gathered}
\rho^{(2)}(\epsilon, T) \simeq \rho_{0}^{(2)}\left(1-\frac{\lambda_{\rho}}{\epsilon_{F} \tau} \ln \left[\frac{1}{2 \tau(|\epsilon|, T)}\right]\right), \\
\rho^{(1)}(\epsilon, T) \simeq \rho_{0}^{(1)}\left(1-\frac{\lambda_{\rho}}{\sqrt{2 \tau(|\epsilon|, T)}}\right) .
\end{gathered}
$$

Consideration of the direct process in diffusion channel depicted in Fig. 1(b) yields similar expression for the DOS. Thus, the $\lambda_{\rho}$ in Eq. (13) should be replaced by $\lambda_{\rho}^{D}$ $=\rho_{0}^{(d)}\left[V(0,0)-\overline{2 V\left(\mathbf{p}^{\prime}-\mathbf{p}^{\prime \prime}, 0\right)}\right]$, where the bar over Coulomb potential corresponding to the Hartree diagram denotes averaging over the Fermi surface and the factor of two appearing because electrons with both spin orientations contribute to the Hartree correction. Note that Hartree term involves zero energy and large momentum transfers.

In above expressions the constant $\lambda_{\rho}$ is the only unknown parameter that cannot be derived in a general way. For a dynamically screened Coulomb interaction within some approximations, the $\lambda_{\rho}$ can be cast into the following form:

$$
\begin{aligned}
\lambda_{\rho} & =\frac{1}{2} \ln \left[\frac{(|\epsilon|, T)}{\hbar \tau\left(D \kappa_{2}^{2}\right)^{2}}\right]-\frac{3}{2} F, \quad(d=2) \\
& =\frac{a \kappa_{3}}{\sqrt{\pi}} \ln ^{1 / 2}\left[\frac{D \kappa_{3}^{2}}{(|\epsilon|, T)}\right]-\frac{3}{2} F, \quad(d=1),
\end{aligned}
$$

where $\kappa_{3}=\sqrt{4 \pi e^{2} \rho_{0}^{(3)}}$ and the first terms represent the exchange contribution to the effective interaction constant $\lambda_{\rho}$ in diffusion channel while the second term $\left(\frac{3}{2} F\right)$ is associated with the Hartree contribution in the same channel. The specific nature for Coulomb interaction in low-dimensional systems manifest itself only in a logarithmic dependence of the constant $\lambda_{\rho}$ on $\epsilon$ and $T$. In contrast to exchange process the Hartree or direct contribution to the $\lambda_{\rho}$ is relatively small $(F \ll 1)$; in both dimensions the evident expression of the parameter $F$ is logarithmic ${ }^{52}$

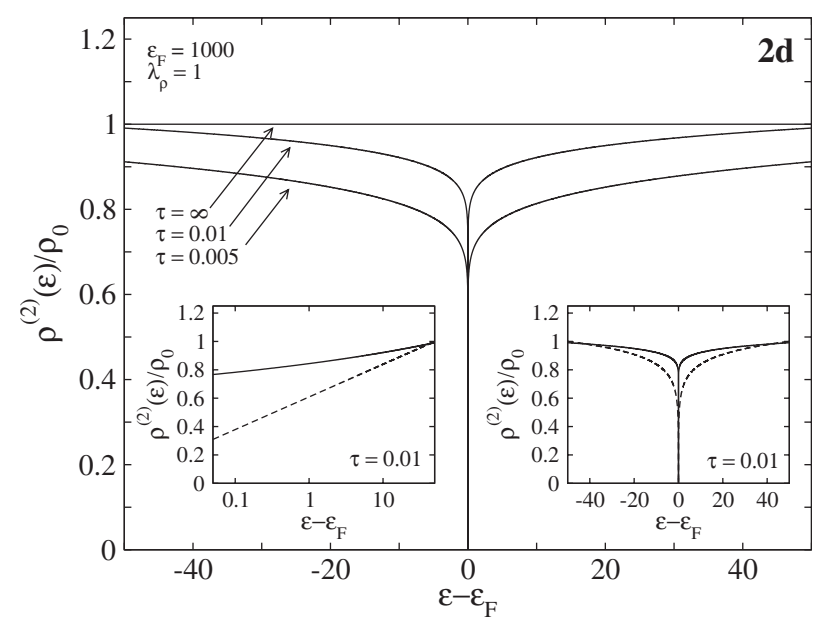

FIG. 3. Normalized DOS of a 2D disordered system as a function of the energy reckoned from the Fermi level at $T=0$ for selected impurity concentrations. The normalized pure DOS $\rho_{0}^{(2)}$, corresponding to $\tau=\infty$, is also given for comparison. In the inset we show the comparison of the DOS with the AAL theory (broken line) in logarithmic (left-hand side) and linear scales (right-hand side) for $\tau=0.01$. Notice the deviations from the logarithmic behavior (broken line).

$$
\begin{aligned}
F & =\frac{2}{x^{2}} \ln \left(1+x^{2}\right), \quad(d=2) \\
& =\frac{1}{\pi \sqrt{x^{2}-1}} \ln \left[\frac{x+\sqrt{x^{2}-1}}{x-\sqrt{x^{2}-1}}\right], \quad(d=1),
\end{aligned}
$$

where $x=\frac{2 p_{F}}{\kappa_{3,2}}$. If $\kappa_{3,2} \ll p_{F}$ then $F \ll 1$. For a detailed discussion the reader is referred to the review paper by Altshuler and Aronov. ${ }^{3}$

So far we have restricted our attention here to the calculation of self-energies in the diffusion channel only. Firstorder corrections to the self-energy given in Fig. 1 include interactions in the Cooperon channel as well. The extension to include the contribution of the Cooperon channel is straightforward. It is clear that the additional self-energy parts from this channel only renormalize the dimensionless interaction constant $\lambda_{\rho}$. Then, one can write the total selfenergy $\Sigma\left(\mathbf{p}, i \epsilon_{n}\right)$ by adding the self-energies in diffusion $\Sigma^{D}\left(\mathbf{p}, i \epsilon_{n}\right)$ and Cooperon $\Sigma^{C}\left(\mathbf{p}, i \epsilon_{n}\right)$ channels as $\Sigma\left(\mathbf{p}, i \epsilon_{n}\right)$ $=\Sigma^{D}\left(\mathbf{p}, i \epsilon_{n}\right)+\Sigma^{C}\left(\mathbf{p}, i \epsilon_{n}\right)$. Accordingly, renormalized dimensionless interaction constant becomes $\lambda_{\rho} \Rightarrow \lambda=\lambda_{\rho}^{D}+\lambda_{\rho}^{C}$, where $\lambda_{\rho}^{C}$ is the interaction constant related to the self-energies in the Cooperon channel in Fig. 1. Consequently, replacing $\lambda_{\rho}$ in Eqs. (15) and (16) by $\lambda$, we obtain the total contribution (including both Cooperon and diffusion channels) to the DOS.

In Figs. 3 and 4 we present normalized DOS near the Fermi energy for 2D and quasi-1D systems at $T=0$ for selected impurity concentrations. In the insets a comparison with the AAL theory is given. As seen, in contrast to firstorder perturbation theory, we obtain a vanishing DOS at Fermi level within the high-order perturbation theory, i.e., in both dimensions $\rho(\epsilon, T=0) \rightarrow 0$ for $\epsilon \rightarrow 0$. This implies that interacting disordered 2D and quasi-1D systems are in insu- 




FIG. 4. Same as Fig. 3 for quasi-1D systems.

lating state at $T=0$ in the diffusive regime. On the other hand, the suppression of the DOS around Fermi level is less singular compared to first-order perturbation theory; for example, in 2D systems the calculated DOS substantially deviates from the logarithmic behavior (see Fig. 3). Such behavior might be connected with the excluded high-order selfenergy diagrams in derivation of Eqs. (15) and (16). Note that the complexity of the diagrams increases dramatically with increasing order of the perturbation theory (for example, see Fig. 2 for second-order self-energy contributions) and those neglected terms can further decrease the DOS around Fermi level. However, these complex terms cannot be systematically included in our treatment. It is worth mentioning that within the present approach the results of the AAL theory is recovered at small corrections, i.e., when $\epsilon_{F} \tau \gg 1$ [see Eqs. (17) and (18)]. Note that in low-dimensional systems the AAL theory is valid as long as corrections to the DOS are small. When corrections to the DOS are so large, one expects the perturbation calculation to break down and the DOS at the Fermi level diverges to negative infinity.

Finally we should note that, despite good agreement between present results and nonperturbative studies for the energy and temperature dependences of the DOS at small corrections (higher energies), an essential difference appears in the asymptotic energy dependence of the DOS. For example in 2D systems [see Eq. (15)] our calculated zero-temperature DOS vanishes as $\rho^{(2)}(\epsilon) \sim[-\ln (\tau|\epsilon|)]^{-1 / 2}$ for $\epsilon \rightarrow 0$, whereas nonperturbative schemes give a power-law behavior. ${ }^{40,47,48}$

\section{ZERO-BIAS ANOMALY OF THE TUNNELING CONDUCTIVITY}

The singularity in the energy dependence of the oneparticle DOS would be reflected in thermodynamic and transport properties of disordered conductors. A clear manifestation of this effect is the minimum of the tunneling conductivity at zero bias. The conductivity of the tunneling contact is related to DOS by

$$
\begin{aligned}
\frac{\sigma^{(d)}(V, T)}{\sigma_{0}^{(d)}}= & \frac{1}{4 T} \int_{-\infty}^{+\infty} d \epsilon \frac{\rho^{(d)}(\epsilon, T)}{\rho_{0}^{(d)}}\left[\frac{1}{\cosh ^{2}\left(\frac{\epsilon-e V}{2 T}\right)}\right. \\
& \left.-\frac{1}{\cosh ^{2}\left(\frac{\epsilon+e V}{2 T}\right)}\right],
\end{aligned}
$$

with $\sigma_{0}$ being classical conductivity, called Drude expression. At zero temperature Eq. (21) reduces to

$$
\frac{\sigma^{(d)}(V)}{\sigma_{0}^{(d)}}=\frac{\rho^{(d)}(e V)}{\rho_{0}^{(d)}} .
$$

As seen from Eq. (22) at $T=0$, the $\sigma(V)$ is directly proportional to DOS; thus the measurement of the tunneling conductivity as a function of bias voltage provides important information on the energy dependence of the one-particle DOS. On the other hand, at finite temperatures Eq. (21) can be written as

$$
\frac{\sigma^{(d)}(T)}{\sigma_{0}^{(d)}}=\frac{2 \rho^{(d)}(T)}{\rho_{0}^{(d)}} \int_{0}^{1} \frac{d x}{\cosh ^{2} x}+\frac{2}{\rho_{0}^{(d)}} \int_{1}^{\infty} \frac{\rho^{(d)}(2 T x)}{\cosh ^{2} x} d x .
$$

In this expression, the major contribution comes from the first integral, and calculation for $2 \mathrm{D}$ and quasi-1D systems gives

$$
\begin{aligned}
\frac{\sigma^{(d)}(T)}{\sigma_{0}^{(d)}} & =\frac{2 C_{0}}{\sqrt{1+\frac{2 \lambda_{\rho}}{\epsilon_{F} \tau} \ln \left(\frac{1}{2 \tau T}\right)}}, \quad(d=2) \\
& =\frac{2 C_{0}}{\sqrt{1+\frac{2 \lambda_{\rho}}{\sqrt{2 \tau T}}}}, \quad(d=1),
\end{aligned}
$$

where $C_{0} \simeq 1$ is a coefficient. Expression (24) shows the change in the tunneling conductivity of the low-dimensional systems corresponding to the temperature in the small value of the potential $(V \rightarrow 0)$.

Since early 1980s several research groups have performed tunneling measurements of quasi-2D disordered metal and semimetal films, and observed the predicted logarithmic dependence of tunneling conductance with voltage. ${ }^{21-25}$ Note however that the experimental techniques used in these early studies were not capable of detecting tunneling current for small voltages. Access to such regimes becomes possible only recently with TDCS method as we mentioned in Sec. I. It should be emphasized that TDCS is unique in allowing complete extraction of the tunneling spectrum of lowdimensional systems. Using this technique Chan and co-workers ${ }^{37,38}$ measured the entire voltage dependence of the tunneling conductance of a $2 \mathrm{D}$ electron system in a $\mathrm{GaAs} / \mathrm{AlGaAs}$ heterostructure for various electron densities. The authors observed the expected logarithmic Coulomb anomaly only in the case of a small suppression of the tunneling current $\left(\epsilon_{F} \tau \gg 1\right)$. However, for large suppressions corresponding to small electron densities for which $\epsilon_{F} \tau \sim 1$, the functional form of the ZBA vs bias voltage was significantly deviating from the predictions of the AAL theory, es- 
pecially in the regime of very small voltages. Furthermore, application of magnetic field perpendicular to the 2D plane results in a linear dependence of the tunneling conductance on voltage near zero bias for all magnetic-field strengths and electron densities. This latter phenomena is not yet completely understood. ${ }^{37}$ Peculiar behavior of the tunneling conductivity at small electron densities can be qualitatively explained by the present theory. Indeed, as seen from Fig. 3 the DOS of 2D systems strongly deviates from the logarithmic behavior for small energies in agreement with observations of Chan and co-workers. ${ }^{37,38}$ However, in our case the deviations seem to be stronger than the one observed in the experiment. As commented in the previous section, this might be due to excluded high-order self-energy diagrams in calculation of the corrections to the DOS.

In contrast to $2 \mathrm{D}$ systems, $\mathrm{ZBA}$ in quasi-1D conductors received less attention. White et al. ${ }^{53}$ reported the first systematic study of the corrections to the DOS of quasi-1D granular aluminum wires. The obtained corrections to the DOS somehow do not have the $V^{-1 / 2}$ dependence predicted by AAL theory but are significantly larger than the corrections observed in corresponding bulk samples. Pierre et al. ${ }^{54}$ measured the tunneling DOS of a metallic wire in perturbative regime in a controlled way and obtained the predicted behavior for the suppression of the tunneling conductance. Recently, Yu and Natelson ${ }^{55}$ studied the ZBA in electrochemically fabricated disordered nanojunctions of various size. For large junctions the authors obtained a small ZBA which is consistent with the perturbative theory of AAL. ${ }^{55}$ However, in atomic scale junctions the observed ZBA was approaching $100 \%$ conductance suppression as $T \rightarrow 0 .{ }^{56}$

Finally, we will briefly discuss the ZBA observed in doped multiwall carbon nanotubes (MWCNT). MWCNT constitute a quasi-1D systems with fascinating physical properties. Several experiments have demonstrated that the charge transport in these systems is diffusive, ${ }^{57-60}$ i.e., showing typical weak localization features in the magnetoconductance and thus, their physical properties show Fermi-liquid (FL) behavior. However, the functional form of the observed ZBA in MWCNT is characteristics of the Luttinger-liquid (LL) state in 1D clean (ballistic) systems of interacting electrons. One of the main features of the LL state in 1D is the power-law dependence of physical quantities, for instance, tunneling DOS, as a function of energy or temperature $\left(\rho(\epsilon) \sim \epsilon^{\alpha}\right) .{ }^{61}$ In MWCNT the observed values for the exponent $\alpha$ is rather scattered between 0.04 and 0.37 depending on the geometry of the samples. ${ }^{62-68}$ The observed peculiar behavior of the tunneling conductivity was attributed to the disorder enhanced $e e$ interaction effects and its theoretical descriptions was beyond the first-order perturbation theory due to large suppressions of the tunneling conductance. Thus, a nonperturbative treatment has recently been put forward by Egger and Gogolin. ${ }^{69}$ The authors predicted a geometry dependent LL-like ZBA in doped MWCNT. Somehow the situation is not so different within present scheme; it follows from Eq. (16) that the tunneling DOS around Fermi level for quasi-1D systems presents a power-law behavior $\left[\rho^{(1)}(\epsilon) \sim(\epsilon \tau)^{1 / 4}\right]$ with an exponent $\alpha=0.25$. Note that, in several tunneling experiments on doped MWCNT, the observed value of $\alpha$ is close to 0.25 in good agreement with our predictions. ${ }^{62,67,68}$

\section{CONCLUSIONS}

In conclusion, we propose a diagrammatic approach to study critical behavior of the one-particle DOS of lowdimensional disordered metals in diffusive regime. By a geometric resummation of the most singular first-order selfenergy corrections via the Dyson equation, we obtain a nondivergent solution for the DOS at low energies while for higher energies the well-known Altshuler-Aronov corrections are recovered. At the Fermi level $\rho^{(d)}(\epsilon, T=0) \rightarrow 0$, this indicates that interacting disordered $2 \mathrm{D}$ and quasi-1D systems are in insulating state at zero temperature. However, asymptotic energy dependence of the calculated DOS differs from those obtained by nonperturbative methods. For 2D systems at zero temperature the DOS vanishes as $\rho^{(2)}(\epsilon) \sim[$ $-\ln (|\epsilon| \tau)]^{-1 / 2}$ for $\epsilon \rightarrow 0$, whereas nonperturbative schemes give a power-law behavior (see Refs. 40, 47, and 48). In contrast to $2 \mathrm{D}$ case, a power-law behavior $\left[\rho^{(1)}(\epsilon) \sim(\epsilon \tau)^{1 / 4}\right]$ is predicted for the asymptotic energy dependence of the DOS of quasi-1D systems. The obtained results are in good agreement with recent tunneling experiments on twodimensional GaAs/AlGaAs heterostructures and quasi-onedimensional doped multiwall carbon nanotubes.

\footnotetext{
*e.sasioglu@fz-juelich.de

†scaliskan@fatih.edu.tr

\#mkumru@fatih.edu.tr

${ }^{1}$ A. A. Abrikosov, L. P. Gor'kov, and I. E. Dzyaloskinskii, Methods of Quantum Field Theory in Statistical Physics (Pergamon, New York, 1965).

${ }^{2}$ G. Bergmann, Phys. Rep. 107, 1 (1984).

${ }^{3}$ B. L. Altshuler and A. G. Aronov, in Electron-Electron Interactions in Disordered Systems, edited by A. L. Efros and M. Pollak (Elsevier, New York, 1985), Chap. 1.

${ }^{4} \mathrm{H}$. Fukuyama, in Electron-Electron Interactions in Disordered Systems, edited by A. L. Efros and M. Pollak (Elsevier, New York, 1985), Chap. 2.

${ }^{5}$ P. A. Lee and T. V. Ramakrishnan, Rev. Mod. Phys. 57, 287 (1985).
}

${ }^{6}$ B. I. Shklovskii and A. L. Efros, Electronic Properties of Doped Semiconductors (Springer, New York, 1984).

${ }^{7}$ J. G. Massey and M. Lee, Phys. Rev. Lett. 75, 4266 (1995).

${ }^{8}$ J. G. Massey and M. Lee, Phys. Rev. Lett. 77, 3399 (1996).

${ }^{9}$ V. Yu. Butko, J. F. DiTusa, and P. W. Adams, Phys. Rev. Lett. 84, 1543 (2000).

${ }^{10}$ B. L. Altshuler, A. G. Aronov, and P. A. Lee, Phys. Rev. Lett. 44, 1288 (1980).

${ }^{11}$ B. L. Altshuler and A. G. Aronov, Sov. Phys. JETP 50, 968 (1979).

${ }^{12}$ Gábor Zala, B. N. Narozhny, and I. L. Aleiner, Phys. Rev. B 64, 214204 (2001); 64, 201201(R) (2001); 65, 020201(R) (2001).

${ }^{13}$ S. A. Vitkalov, K. James, B. N. Narozhny, M. P. Sarachik, and T. M. Klapwijk, Phys. Rev. B 67, 113310 (2003).

${ }^{14}$ I. Paul, C. Pépin, B. N. Narozhny, and D. L. Maslov, Phys. Rev. 
Lett. 95, 017206 (2005).

${ }^{15}$ E. McCann, K. Kechedzhi, Vladimir I. Fal'ko, H. Suzuura, T. Ando, and B. L. Altshuler, Phys. Rev. Lett. 97, 146805 (2006).

${ }^{16}$ Y. Adamov, I. V. Gornyi, and A. D. Mirlin, Phys. Rev. B 73, 045426 (2006); I. V. Gornyi and A. D. Mirlin, ibid. 69, 045313 (2004); Ya. M. Blanter and A. D. Mirlin, ibid. 57, 4566 (1998).

${ }^{17}$ E. G. Mishchenko, A. V. Andreev, and L. I. Glazman, Phys. Rev. Lett. 87, 246801 (2001); A. M. Rudin, I. L. Aleiner, and L. I. Glazman, Phys. Rev. B 55, 9322 (1997); L. Shekhtman and L. I. Glazman, ibid. 52, R2297 (1995).

${ }^{18}$ E. Kogan and B. Rosenstein, Phys. Rev. B 69, 113105 (2004).

${ }^{19}$ A. A. Koulakov, Phys. Rev. B 62, 6858 (2000).

${ }^{20}$ W. L. McMillan and Jack Mochel, Phys. Rev. Lett. 46, 556 (1981).

${ }^{21}$ Y. Imry and Z. Ovadyahu, Phys. Rev. Lett. 49, 841 (1982).

${ }^{22}$ M. E. Gershenson, V. N. Gubankov, and M. I. Fale $\check{1}$, JETP Lett. 41, 534 (1985).

${ }^{23}$ A. E. White, R. C. Dynes, and J. P. Garno, Phys. Rev. B 31, 1174 (1985).

${ }^{24}$ J. M. Valles, Jr., R. C. Dynes, and J. P. Garno, Phys. Rev. B 40, 7590 (1989).

${ }^{25}$ Shih-Ying Hsu and J. M. Valles, Phys. Rev. B 49, 16600 (1994).

${ }^{26}$ S. V. Kravchenko, G. V. Kravchenko, J. E. Furneaux, V. M. Pudalov, and M. D'Iorio, Phys. Rev. B 50, 8039 (1994); S. V. Kravchenko, W. E. Mason, G. E. Bowker, J. E. Furneaux, V. M. Pudalov, and M. D'Iorio, ibid. 51, 7038 (1995).

${ }^{27}$ S. V. Kravchenko, D. Simonian, M. P. Sarachik, Whitney Mason, and J. E. Furneaux, Phys. Rev. Lett. 77, 4938 (1996); D. Simonian, S. V. Kravchenko, M. P. Sarachik, and V. M. Pudalov, ibid. 79, 2304 (1997); S. V. Kravchenko and T. M. Klapwijk, ibid. 84, 2909 (2000).

${ }^{28}$ H. B. Chan, P. I. Glicofridis, R. C. Ashoori, and M. R. Melloch, Phys. Rev. Lett. 79, 2867 (1997).

${ }^{29}$ E. Abrahams, S. V. Kravchenko, and M. P. Sarachik, Rev. Mod. Phys. 73, 251 (2001).

${ }^{30}$ S. V. Kravchenko and M. P. Sarachik, Rep. Prog. Phys. 67, 1 (2004).

${ }^{31}$ Qimiao Si and C. M. Varma, Phys. Rev. Lett. 81, 4951 (1998).

${ }^{32}$ B. L. Altshuler and D. L. Maslov, Phys. Rev. Lett. 82, 145 (1999).

${ }^{33}$ E. P. Nakhmedov, V. Prigodin, S. Çalışkan, and E. Şaşıŏlu, Phys. Rev. B 66, 233105 (2002); E. P. Nakhmedov and V. N. Prigodin, ibid. 65, 235107 (2002).

${ }^{34}$ E. P. Nakhmedov and K. Morawetz, Phys. Rev. B 66, 195333 (2002); E. P. Nakhmedov, K. Morawetz, M. Ameduri, A. Yurtsever, and C. Radehaus, ibid. 67, 205106 (2003).

${ }^{35}$ B. Rosenstein and Tran Minh-Tien, Phys. Rev. B 68, 245321 (2003).

${ }^{36}$ M. S. Foster and A. W. W. Ludwig, Phys. Rev. B 74, 241102(R) (2006); 77, 165108 (2008).

${ }^{37}$ Ho Bun Chan, Ph.D. thesis, MIT, 1999.

${ }^{38}$ R. C. Ashoori and H. B. Chan, Solid State Commun. 127, 79 (2003).

${ }^{39}$ O. E. Dial, R. C. Ashoori, L. N. Pfeiffer, and K. W. West, Nature (London) 448, 176 (2007).

${ }^{40}$ A. M. Finkelstein, Zh. Eksp. Teor. Fiz. 84, 168 (1983) [Sov. Phys. JETP 57, 97 (1983)].

${ }^{41}$ R. Oppermann, Z. Phys. B: Condens. Matter 49, 273 (1983).

${ }^{42}$ H. Fukuyama, Y. Isawa, and H. Yasuhara, J. Phys. Soc. Jpn. 52,
16 (1983).

${ }^{43}$ C. Castellani, C. Di Castro, and G. Forgacs, Phys. Rev. B 30, 1593 (1984).

${ }^{44}$ D. Belitz and T. R. Kirkpatrick, Phys. Rev. B 48, 14072 (1993); D. Belitz and T. R. Kirkpatrick, Rev. Mod. Phys. 66, 261 (1994).

${ }^{45}$ V. Dobrosavljević, Elihu Abrahams, E. Miranda, and Sudip Chakravarty, Phys. Rev. Lett. 79, 455 (1997).

${ }^{46}$ S. Levitov and A. V. Shytov, Zh. Eksp. Teor. Fiz. 66, 200 (1997) [JETP Lett. 66, 214 (1997)].

${ }^{47}$ P. Kopietz, Phys. Rev. Lett. 81, 2120 (1998); L. Bartosch and P. Kopietz, Eur. Phys. J. B 28, 29 (2002).

${ }^{48}$ A. Kamenev and A. Andreev, Phys. Rev. B 60, 2218 (1999).

${ }^{49}$ Jörg Rollbühler and Hermann Grabert, Phys. Rev. Lett. 87, 126804 (2001)

${ }^{50}$ Yuval Oreg, P. W. Brouwer, B. D. Simons, and Alexander Altland, Phys. Rev. Lett. 82, 1269 (1999).

${ }^{51}$ E. P. Nakhmedov, M. Kumru, and R. Oppermann, Phys. Rev. Lett. 84, 3930 (2000); E. P. Nakhmedov, H. Feldmann, R. Oppermann, and M. Kumru, Phys. Rev. B 62, 13490 (2000).

${ }^{52}$ T. F. Rosenbaum, K. Andres, G. A. Thomas, and P. A. Lee, Phys. Rev. Lett. 46, 568 (1981).

${ }^{53}$ Alice E. White, R. C. Dynes, and J. P. Garno, Phys. Rev. Lett. 56, 532 (1986).

${ }^{54}$ F. Pierre, H. Pothier, P. Joyez, Norman O. Birge, D. Esteve, and M. H. Devoret, Phys. Rev. Lett. 86, 1590 (2001).

${ }^{55}$ L. H. Yu and D. Natelson, Phys. Rev. B 68, 113407 (2003).

${ }^{56}$ L. H. Yu and D. Natelson, Appl. Phys. Lett. 82, 2332 (2003).

${ }^{57}$ L. Langer, V. Bayot, E. Grivei, J.-P. Issi, J. P. Heremans, C. H. Olk, L. Stockman, C. Van Haesendonck, and Y. Bruynseraede, Phys. Rev. Lett. 76, 479 (1996).

${ }^{58}$ A. Bachtold, C. Strunk, J.-P. Salvetat, J.-M. Bonard, L. Forro, T. Nussbaumer, and C. Schonenberger, Nature (London) 397, 673 (1999).

${ }^{59}$ A. Bachtold, M. S. Fuhrer, S. Plyasunov, M. Forero, Erik H. Anderson, A. Zettl, and Paul L. McEuen, Phys. Rev. Lett. 84, 6082 (2000).

${ }^{60}$ R. Tarkiainen, M. Ahlskog, A. Zyuzin, P. Hakonen, and M. Paalanen, Phys. Rev. B 69, 033402 (2004).

${ }^{61}$ C. L. Kane and Matthew P. A. Fisher, Phys. Rev. B 46, 15233 (1992).

${ }^{62}$ A. Bachtold, M. de Jonge, K. Grove-Rasmussen, P. L. McEuen, M. Buitelaar, and C. Schönenberger, Phys. Rev. Lett. 87, 166801 (2001)

${ }^{63}$ E. Graugnard, P. J. de Pablo, B. Walsh, A. W. Ghosh, S. Datta, and R. Reifenberger, Phys. Rev. B 64, 125407 (2001).

${ }^{64}$ R. Tarkiainen, M. Ahlskog, J. Penttilä, L. Roschier, P. Hakonen, M. Paalanen, and E. Sonin, Phys. Rev. B 64, 195412 (2001).

${ }^{65}$ K. Liu, P. Avouris, R. Martel, and W. K. Hsu, Phys. Rev. B 63, 161404(R) (2001).

${ }^{66}$ W. Yi, L. Lu, H. Hu, Z. W. Pan, and S. S. Xie, Phys. Rev. Lett. 91, 076801 (2003).

${ }^{67}$ V. Krstić, S. Blumentritt, J. Muster, S. Roth, and A. Rubio, Phys. Rev. B 67, 041401(R) (2003).

${ }^{68}$ A. Kanda, K. Tsukagoshi, Y. Aoyagi, and Y. Ootuka, Phys. Rev. Lett. 92, 036801 (2004).

${ }^{69}$ R. Egger and A. O. Gogolin, Phys. Rev. Lett. 87, 066401 (2001). 